\title{
Research Progress of Artemisinin in Treating Chronic Kidney Disease
}

\author{
Lanyue Xiong,"\#, Yang Chen ${ }^{2, \#, ~ J u e l i q i ~ W a n g ², ~ Y i x i u ~ Y a n g ~}{ }^{2}$, Heyong Wang,* \\ ${ }^{1}$ Chengdu First Peoples Hosp, Dept Cardiol, 18 North Wanxiang Rd, Chengdu 610016, Sichuan, P R China \\ ${ }^{2}$ Sichuan Hospital of Integrated Chinese and Western Medicine, Chengdu 610042, Sichuan, PR China \\ ${ }^{3}$ School of Basic Medical Sciences, Chengdu University of Traditional Chinese Medicine, Chengdu 611137, Sichuan, PR China \\ \#These authors have contributed equally to this work and are co-first authors \\ *Correspondence Author
}

\begin{abstract}
Artemisinin and its derivatives are not only used to treat malaria, but more and more animal experiments have shown that artemisinin and its derivatives have therapeutic effects on kidney diseases, and can be used to treat various kidney diseases, such as systemic lupus erythematosus, IgA nephropathy, diabetic nephropathy, renal fibrosis, etc. And its toxic and side effects are small. The main adverse reactions were rash and allergy. The application of artemisinin and its derivatives in renal diseases has been widely studied and further promoted, and its mechanism of action, clinical and pharmacological research need to be further deepened.
\end{abstract}

Keywords: Artemisinin (Art), Chronic kidney disease, Immune regulation, Nephrotic syndrome, IgA nephropathy, Membranous nephropathy, Diabetic nephropathy, Lupus nephritis, Hypertensive nephropathy, Renal fibrosis.

\section{Introduction}

Both acute kidney injury (AKI) and chronic kidney disease (CKD) that lead to diminished kidney function are interdependent risk factors for increased mortality. If untreated over time, end-stage renal disease (ESRD) is an inevitable outcome[1]. The imbalance between the molecular mechanisms that govern oxidative stress, inflammation, immunity, and cell death are important causes of acute kidney injury (AKI) and chronic kidney diseases (CKD)[1]. Both AKI and CKD can lead to diminished kidney function and are associated with high mortality and morbidity. Accumulated evidence demonstrated that natural products are alternative sources for treating renal diseases on account of the conventional experience and multi-target characteristics[2].

"Discovery of artemisinin: a gift of traditional Chinese medicine to the world," said Tu Youyou, Nobel Prize winner. Artemisinin and its derivatives were mainly used to treat malaria in the early stage, and later they were also widely used in rheumatic immune diseases such as systemic lupus erythematosus. But as Tu Youyou said, "although artemisinin has been discovered for nearly half a century, its deep mechanism still needs to be studied." With the deepening of research, its anti-tumor, anti-infection and immune regulatory functions are gradually prominent, and its application in chronic kidney disease has been paid more and more attention by clinicians and scientific researchers. This paper reviews the research progress of artemisinin and its derivatives in the treatment of chronic kidney diseases in recent years, to provide new clinical and scientific research ideas for the further development and utilization of artemisinin and its derivatives.

\section{Nephrotic Syndrome}

One study was designed to test the therapeutic effect of a new antimalarial drug, artesunate in an experimental model of nephrotic syndrome[3]. The data suggest that artesunate therapy can ameliorate proteinuria, and suppress the progression of glomerular lesions in an experimental model of nephrotic syndrome, it may also be recommended as a lipid-lowering drug.

\section{IgA Nephropathy}

Immunoglobulin A nephropathy (IgAN) is an autoimmune kidney disease with complex pathogenesis leading to end-stage renal damage[4]. The prime pathological characteristics of IgAN are IgA immune complexes deposition accompanied by mesangial cell proliferation and urine protein elevation. In vivo, the results demonstrated that AH could efficiently ameliorate kidney damage by improving kidney dysfunction and reducing the levels of $24 \mathrm{~h}$ urine protein, $\operatorname{IgA}$ and $\operatorname{IgG}$ immune complexes deposition in the glomerulus of IgAN rats. Interestingly, AH obviously promoted the secretion of exosomes in renal tissues, inhibited the expressions of nuclear factor- $\mathrm{KB}(\mathrm{NF}-\mathrm{\kappa B})$ signaling and NLRP3 inflammasome-related proteins, including I $\mathrm{KB}-\alpha$, p-p65, NLRP3, ASC, IL-1 $\beta$ and caspase-1 in IgAN rats. In vitro, further mechanistic study illustrated that exosomes derived from human renal tubular epithelial cells (HK-2) were significantly enhanced by $\mathrm{AH}$, which could be utterly taken up in human mesangial cells (HMCs) and inhibited the activation of NF- $\mathrm{KB}$ pathway and NLRP3 inflammasome after AH intervention. However, GW4869 interdicted the promotive effect of $\mathrm{AH}$ on exosomes from HK-2 cells and the suppression of exosomes on NF-KB/NLRP3 activation in HMCs. Taken together, the study demonstrated that there was an inhibitory effect of AH therapy on NF- $\mathrm{kB} / \mathrm{NLRP} 3$ signaling via mediating exosomes release in IgAN rats, which provided an alternative approach for IgAN treatment.

In another study, renal biopsy specimens were collected for immunohistochemistry[5]. In vitro, $25 \mu \mathrm{g} / \mathrm{ml}$ concentrations of aggregated IgA1 (aIgA1) was used to construct the IgAN mesangial cell model. Stimulated human mesangial cells (HMCs) were treated for $24 \mathrm{~h}$ with DHA $(0-15 \mu \mathrm{M})$ and were collected for western blot analyses. Cell proliferation was assessed by Cell Counting Kit 8 (CCK8) and 3-(4,5-dimethyl -2-thiazolyl)-2,5-diphenyl-2-H-tetrazolium bromide (MTT) assay. In vitro, our results showed that DHA could 
downregulate the mammalian target of rapamycin/ribosomal protein S6 kinase beta-1 (mTOR/S6K1) signaling pathway, promote cell autophagy, and ameliorate cell proliferation in aIgA1-induced HMCs. The results suggested that DHA may represent a novel class of mTOR inhibitor and promote an anti-proliferation effect in IgAN HMCs, which provides an alternative approach for IgAN treatment.

Another study suggested that Artesunate have the ability to downregulate the expression of MCP-1 in renal tissue[6], which may explain one of the mechanisms of Artesunate effectiveness in the clinical treatment of $\operatorname{IgA}$ nephropathy.

\section{Membranous Nephropathy}

Artemisinin analogue SM934 is a novel water-soluble artemisinin derivative with immunoregulatory activities that has been used to treat murine lupus nephritis[7]. In the current study, we investigated the effects of SM934 on rat experimental membranous nephropathy. Treatment of PHN rats with SM934 or prednisolone attenuated the progression of glomerulonephritis and renal fibrosis, as evidenced by the reduced level of proteinuria and circulating antibodies, as well as by the reduced immune complex deposition, reversed podocyte injuries, and attenuated tubulointerstitial fibrosis in the kidneys. Furthermore, the two drugs suppressed TGF- $\beta 1$ expression and $\mathrm{Smad} 2 / 3$ phosphorylation, and increased Smad7 expression in the kidneys. The two doses of SM934 produced almost identical therapeutic effects on PHN rats. Pretreatment with SM934 or a C3a receptor antagonist blocked the $\mathrm{C} 3 \mathrm{a}$-induced epithelial-mesenchymal transition in HK-2 cells in vitro.

\section{Diabetic Nephropathy}

One study constructed an animal model of DN by injection of streptozotocin (STZ) in rats[8]. Then examined the profile of differentially expressed genes following administration of Art using RNA-sequencing (KANGCH\&EN, Shanghai, China). Five genes identified by RNA-sequencing were randomly selected and validated by qRT-PCR. Bioinformatic analyses were performed to study these differentially expressed genes. They identified a total of 31 genes that were significantly up-regulated in DN samples compared to both normal and Art treatment samples, and 38 genes that were significantly down-regulated in DN samples compared to both normal and Art treatment samples. The identified genes were associated with a list of gene ontology (GO) terms and Kyoto Encyclopedia of Genes and Genomes (KEGG) pathways and may be involved in the mechanism underlying Art treatment of DN. The results from the study demonstrate that genes are aberrantly expressed after Art treatment and identify promising targets in the treatment of DN with artemisinin.

One study demonstrated that artemisinin could reduce early renal oxidative stress damage in $\mathrm{DN}$ rats by inhibiting TGF- $\beta 1$ protein expression in kidney tissues as well as activating the Nrf2 signaling pathway and enhancing the expression of antioxidant proteins, thereby exerting the protective effects on DN kidney[9]. The current study is the first report of ATZ on attenuating effects on kidney of DN rats, which could lay solid theoretical foundations on clinical application of ATZ to treat DN.
Another study demonstrated that Artemether significantly could reduce rates of weight gain and fasting blood glucose levels[10], improved islet function and insulin resistance and reduced serum lipid levels to varying degrees in $\mathrm{db} / \mathrm{db}$ mice. Artemether exerted a positive effect on islet vacuolar degeneration and hepatic steatosis, and increased expressions of AMP-activated protein kinase, glucose transporter 4 and Insulin receptor $\beta$ protein in the liver of these $\mathrm{db} / \mathrm{db}$ mice. With the use of liver protein chip detection, we found that artemether significantly improved the immune microenvironment, down-regulated the expression of inflammatory factors and activated the cytokine-mediated signaling pathway through cytokine-cytokine receptor interactions.

Another study demonstrated that Artesunate ameliorates decreased HG-induced the expression levels of toll-like receptor 4 (TLR4), myeloid differentiation primary response gene 88 (MyD88), nuclear factor $\kappa \mathrm{B}(\mathrm{NF}-\kappa \mathrm{B}) \mathrm{p}-\mathrm{p} 65$, and nod-like receptor protein 3 (NLRP3). Inhibition of the TLR4/NF- $\kappa \mathrm{B}$ pathway suppressed NLRP3 inflammasome in HBZY-1 cells[11].

\section{Lupus Nephritis}

Lupus nephritis (LN) is one of the most common and severe manifestations of systemic lupus erythematosus, leading to permanent renal damage and chronic kidney disease.one study was observed that L-HCQ + ART and H-HCQ ameliorated the LN-induced body weight decrease, and significantly decreased the levels of anti-double stranded DNA, antinuclear antibodies, immunoglobulin $\mathrm{G}$, interferon $\gamma$, tumor necrosis factor- $\alpha$ and transforming growth factor- $\beta 1$, as well as improved the kidney and spleen pathology, when compared with the model group. In addition, L-HCQ + ART and H-HCQ treatments induced KLF15 upregulation and $\mathrm{NF}-\kappa \mathrm{B}$ downregulation. These results indicated that treatment with L-HCQ + ART exerted renoprotective effects by regulating the expression levels of cytokines, KLF15 and $\mathrm{NF}-\kappa \mathrm{B}$. This combination treatment may have a similar immunosuppressive effect as PDS and H-HCQ, and may be a promising alternative for LN treatment[12].

High-mobility group box 1 (HMGB1), a nuclear protein with a proinflammatory cytokine activity, binds specifically to TLR4 to induce inflammation. One study develop PEGylated TAT peptide-cationic liposomes (TAT-CLs) to deliver anti-HMGB1 siRNA and dihydroartemisinin (DHA). In vitro, it showed significantly greater uptake compared with unmodified liposomes and significant inhibition of glomerular mesangial cell proliferation. TAT-CLs-DHA/ siRNA inhibited NF- $\mathrm{KB}$ activation in a concentrationdependent manner. Real-time PCR and Western blot analysis showed that TAT-CLs-DHA/siRNA downregulated expression of HMGB1 mRNA and protein. TAT-CLs-DHA/ siRNA markedly diminished Toll-like receptor 4 (TLR4) expression and subsequent activation of MyD88, IRAK4, and NF- $\kappa$ B. TAT-CLs-DHA/siRNA may have the potential for treatment of inflammatory diseases such as LN mediated by the TLR4 signaling pathway[13].

\section{Hypertensive Nephropathy}

Hypertensive nephropathy is one of the important causes of 
chronic kidney disease. One study indicate that treatment with artemisinin has beneficial effects on reducing the heart rate and basal vascular tension and improving endotheliumdependent vascular relaxation in hypertension, which might occur by increasing eNOS activation and NO release and inhibiting NAD(P)H oxidase derived ROS production[14].

\section{Renal Fibrosis}

Renal fibrosis is the most common final stage of progressive renal disease, characterized by fibroblast proliferation, fibroblast-to-myofibroblast differentiation and excessive accumulation of extracellular matrix. Dihydroartemisinin (DHA) exerts antitumor, antibacterial, and antifibrotic effects[15]. From the experiment they found that, In vivo, DHA reduced UUO-induced morphological and pathological changes and the degree of renal fibrosis. In addition, DHA mitigated fibroblast proliferation and differentiation in kidney tissue induced by UUO. In vitro, DHA significantly attenuated the TGF- $\beta 1$-induced primary human kidney fibroblast proliferation and fibroblast-to-myofibroblast differentiation. Moreover, treatment with DHA attenuated the up-regulation of phosphorylation of phosphatidylinositol-3kinase (PI3K) and protein kinase B (AKT) in UUO model and TGF- $\beta 1$-induced primary human kidney fibroblasts.

\section{Conclusion}

Previous studies have shown that artemisinin and its derivatives have many functions, such as anti malaria, anti infection, anti-virus, anti-tumor, immune regulation and so on. And their therapeutic effect in the treatment of rheumatic immune diseases is gradually recognized. It is involved in the regulation of a variety of immune factors (IL-6, IL-10, CD4 T cells), cytokines (TGF-1, Treg cells, PDGF) and signal pathways (NF-B pathway, mTOR signal pathway). The occurrence and development of nephropathy is related to a variety of immune abnormalities. Artemisinin can improve oxidative stress, reduce inflammatory response and inhibit cellular and humoral immunity in a variety of ways. At present, there are many animal experimental evidences that artemisinin and its derivatives have therapeutic effects on chronic kidney disease, but there are few clinical research literatures. In the future, we should deeply study the role of artemisinin and its derivatives in clinical research, so as to better improve the clinical efficacy of chronic kidney disease and give better play to the role of traditional Chinese medicine on human health.

\section{References}

[1] Sureshbabu A, Ryter SW, Choi ME. Oxidative stress and autophagy: crucial modulators of kidney injury. Redox Biol. 2015; 4: 208-14.

[2] Tecklenborg J, Clayton D, Siebert S, Coley SM. The role of the immune system in kidney disease. Clin Exp Immunol. 2018 May; 192(2): 142-150.

[3] Razavi A, Nouri HR, Mehrabian F, Mirshafiey A. Treatment of experimental nephrotic syndrome with artesunate. Int J Toxicol. 2007 Jul-Aug; 26(4): 373-80.

[4] Bai L, Li J, Li H, Song J, Zhou Y, Lu R, Liu B, Pang Y, Zhang $\mathrm{P}$, Chen J, Liu $\mathrm{X}$, Wu J, Liang $\mathrm{C}$, Zhou J.
Renoprotective effects of artemisinin and hydroxychloroquine combination therapy on IgA nephropathy via suppressing NF- $\kappa \mathrm{B}$ signaling and NLRP3 inflammasome activation by exosomes in rats. Biochem Pharmacol. 2019 Nov; 169: 113619.

[5] Xia M, Liu D, Tang X, Liu Y, Liu H, Liu Y, Chen G, Liu H. Dihydroartemisinin inhibits the proliferation of IgAN mesangial cells through the mTOR signaling pathway. Int Immunopharmacol. 2020 Mar; 80: 106125.

[6] Mi XH, Luan S, Ma XY, Ma YX, Zeng Y, Wu WH, Qin W. Effects of Artesunate on MCP-1 and MCP-1 mRNA expression of renal tissue in the rat $\operatorname{IgA}$ nephropathy model. Sichuan Da Xue Xue Bao Yi Xue Ban. 2009 Sep; 40(5): 821-5.

[7] Li TT, Zhang XH, Jing JF, Li X, Yang XQ, Zhu FH, Tang W, Zuo JP. Artemisinin analogue SM934 ameliorates the proteinuria and renal fibrosis in rat experimental membranous nephropathy. Acta Pharmacol Sin. 2015 Feb; 36(2): 188-99.

[8] Xiang M, Chen Z, He L, Xiong G, Lu J. Transcription profiling of artemisinin-treated diabetic nephropathy rats using high-throughput sequencing. Life Sci. 2019 Feb 15; 219: 353-363.

[9] Zhang H, Qi S, Song Y, Ling C. Artemisinin attenuates early renal damage on diabetic nephropathy rats through suppressing TGF- $\beta 1$ regulator and activating the Nrf2 signaling pathway. Life Sci. 2020 Sep 1; 256: 117966.

[10] Fu W, Ma Y, Li L, Liu J, Fu L, Guo Y, Zhang Z, Li J, Jiang H. Artemether Regulates Metaflammation to Improve Glycolipid Metabolism in $\mathrm{db} / \mathrm{db}$ Mice. Diabetes Metab Syndr Obes. 2020 May 19; 13: 1703-1713.

[11] Sun Z, Ma Y, Chen F, Wang S, Chen B, Shi J. Artesunate ameliorates high glucose-induced rat glomerular mesangial cell injury by suppressing the TLR4/NF$\kappa \mathrm{B} / \mathrm{NLRP} 3$ inflammasome pathway. Chem Biol Interact. 2018 Sep 25; 293: 11-19.

[12] Liang N, Zhong Y, Zhou J, Liu B, Lu R, Guan Y, Wang Q, Liang C, He Y, Zhou Y, Song J, Zhou J. Immunosuppressive effects of hydroxychloroquine and artemisinin combination therapy via the nuclear factor- $\kappa \mathrm{B}$ signaling pathway in lupus nephritis mice. Exp Ther Med. 2018 Mar;15(3):2436-2442.

[13] Diao L, Tao J, Wang Y, Hu Y, He W. Co-Delivery of Dihydroartemisinin And HMGB1 siRNA By TAT-Modified Cationic Liposomes Through The TLR4 Signaling Pathway For Treatment Of Lupus Nephritis. Int J Nanomedicine. 2019 Nov 4; 14: 8627-8645.

[14] Liu X, Wang X, Pan Y, Zhao L, Sun S, Luo A, Bao C, Tang H, Han Y. Artemisinin Improves AcetylcholineInduced Vasodilatation in Rats with Primary Hypertension. Drug Des Devel Ther. 2021 Nov 2; 15: 4489-4502.

[15] Zhang B, Liu P, Zhou Y, Chen Z, He Y, Mo M, Dai G, Xia W, Du Y, Liu Y, Chen X. Dihydroartemisinin attenuates renal fibrosis through regulation of fibroblast proliferation and differentiation. Life Sci. 2019 Apr 15; 223: 29-37. 